\title{
POLAR DECOMPOSITION OF SCALE-HOMOGENEOUS MEASURES WITH APPLICATION TO LÉVY MEASURES OF STRICTLY STABLE LAWS
}

\author{
STEVEN N. EVANS AND ILYA MOLCHANOV
}

\begin{abstract}
A scaling on some space is a measurable action of the group of positive real numbers. A measure on a measurable space equipped with a scaling is said to be $\alpha$-homogeneous for some nonzero real number $\alpha$ if the mass of any measurable set scaled by any factor $t>0$ is the multiple $t^{-\alpha}$ of the set's original mass. It is shown rather generally that given an $\alpha$-homogeneous measure on a measurable space there is a measurable bijection between the space and the Cartesian product of a subset of the space and the positive real numbers (that is, a "system of polar coordinates") such that the push-forward of the $\alpha$-homogeneous measure by this bijection is the product of a probability measure on the first component (that is, on the "angular" component) and an $\alpha$-homogeneous measure on the positive half-line (that is, on the "radial" component). This result is applied to the intensity measures of Poisson processes that arise in Lévy-Khinchin-Itô-like representations of infinitely divisible random elements. It is established that if a strictly stable random element in a convex cone admits a series representation as the sum of points of a Poisson process, then it necessarily has a LePage representation as the sum of i.i.d. random elements of the cone scaled by the successive points of an independent unit intensity Poisson process on the positive half-line each raised to the power $-\frac{1}{\alpha}$.
\end{abstract}

\section{INTRODUCTION}

One may consider infinitely divisible random elements in very general settings where there is a binary operation on the carrier space such that it makes sense to speak of the "addition" of two random elements.

Date: September 15, 2018.

2010 Mathematics Subject Classification. 28A50, 28C10, 60B15, 60E07.

Key words and phrases. disintegration; infinite divisibility; LePage representation.

SNE supported in part by NSF grant DMS-09-07630 and NIH grant 1R01GM109454-01. IM supported in part by Swiss National Science Foundation grants 200021-137527 and 200021-153597. 
Once one rules out pathologies such as random elements with idempotent distributions (that is, probability measures that are equal to their convolution with themselves), the main step towards understanding infinitely divisible random elements usually consists of establishing their representation as a sum of the points of a Poisson random measure and possibly also constant terms and random elements with Gaussian-like distributions. Results of this type start from the classical Lévy-Khinchin theorem for Euclidean space, with further extensions to Banach spaces [1, 25], groups [22], function spaces [2, 13, 23], and general semigroups [26].

A convex cone is a semigroup equipped with a scaling, that is, with an action of the group of positive real numbers that interacts suitably with the semigroup operation. There is a natural notion of stability for random elements of such a carrier space that extends the usual notion of stability for random elements of Euclidean space, see [5]. For strictly stable random elements, the intensity measure of the corresponding Poisson process - the Lévy measure of the random element - is scalehomogeneous, that is, its value on any set scaled by any positive real number equals the value on the original set up to a factor given by a (fixed) power of the scaling constant. A scale-homogeneous measure on $\mathbb{R}^{d}$ that is finite outside a neighborhood of the origin can be represented in polar coordinates as the product of a probability measure on the unit sphere (the directional or angular component) and a scale-homogeneous measure on the positive reals (the radial component). A nontrivial scale-homogeneous measures on the positive reals that is finite outside neighborhoods of the origin is necessarily of the form $c \alpha t^{-(\alpha+1)} d t$ for some constants $c>0$ and $\alpha>0$. The unit sphere need not be the unit sphere for the Euclidean norm: the unit sphere for any norm on $\mathbb{R}^{d}$ serves equally well. The situation becomes more complicated for more general spaces where there may be no natural candidates to play the rôle of the unit sphere.

Such a polar decomposition of the Lévy measure leads to a representation of stable random elements as the sum of a series obtained from a sequence of i.i.d. random elements scaled by the points of a Poisson process on the positive real line with intensity measure of the form $\alpha t^{-(\alpha+1)} d t$ or, equivalently by points of the form $\Gamma_{k}^{-\frac{1}{\alpha}}$, where $\left(\Gamma_{k}\right)_{k \in \mathbb{N}}$ are the points of a unit intensity Poisson process on the positive real line. Such a representation was first obtained in [16] for Euclidean space and since then it and its various extensions have been called the LePage series representation. For example, such a representation was given for random elements of a suitable space of functions with 
the semigroup operation being addition in [27] and with the semigroup operation being maximum in [6].

Section 2 presents the main result that concerns a polar decomposition of scale-homogeneous measures on quite general spaces. Proposition 2.8 is inspired by the argument used in [9, Th. 10.3] to derive the LePage series representation for stable metric measure spaces. A similar rescaling argument was used in the context of max-stable sequences in [6].

In Section 3 we review the definition of a convex cone, introduce stable random elements on such carrier spaces, and apply the general decomposition results to derive the series representation of strictly stable random elements in convex cones. Such a series representation is a consequence of the polar decomposition of the Lévy measure and expresses any strictly stable random element as a sequence of i.i.d. random elements scaled by a power of the successive points of the unit intensity Poisson point process on the positive half line. The key hypotheses we require are a one-sided continuity property of bounded semicharacters and the existence of a measurable transversal for the action of the positive real numbers on the carrier space.

Section 4 presents several applications, some in well-known settings and others in more novel contexts, where our approach streamlines the derivation of the LePage series representation of a stable random elements.

\section{Decomposition of SCALE-Homogeneous measures}

Definition 2.1. Let $\mathbb{X}$ be a measurable space with a $\sigma$-algebra $\mathfrak{B}$, and let $(x, t) \mapsto t x \in \mathbb{X}$ be a pairing of $x \in \mathbb{X}$ and $t \in \mathbb{R}_{++}:=(0, \infty)$ which is $\mathfrak{B} \otimes \mathcal{B}\left(\mathbb{R}_{++}\right) / \mathfrak{B}$-measurable, where $\mathcal{B}\left(\mathbb{R}_{++}\right)$is the Borel $\sigma$-algebra on $\mathbb{R}_{++}$. Assume that this pairing is an action of the group $\mathbb{R}_{++}$(where the group operation is the usual multiplication of real numbers) on $\mathbb{X}$; that is, for all $t, s>0$ and $x \in \mathbb{X}, t(s x)=(t s) x$ and $1 x=x$. We refer to such a pairing as a scaling.

Remark 2.2. For $B \in \mathfrak{B}$ and $t \in \mathbb{R}_{++}$, set $t B:=\{t x: x \in B\}$. The measurability of the map $x \mapsto t x$ for each $t \in \mathbb{R}_{++}$yields that $s B=\left\{x:\left(s^{-1}\right) x \in B\right\} \in \mathfrak{B}$ for all $B \in \mathfrak{B}$ and $s \in \mathbb{R}_{++}$.

Definition 2.3. A measure $\nu$ on $\mathbb{X}$ is said to be $\alpha$-homogeneous for some $\alpha \in \mathbb{R} \backslash\{0\}$ if

$$
\nu(s B)=s^{-\alpha} \nu(B), \quad B \in \mathfrak{B}, s>0 .
$$

Remark 2.4. By redefining the pairing to be $(x, t) \mapsto t^{-1} x$, we may assume that $\alpha>0$ and we will do so from now on. Moreover, by 
redefining the pairing to be $(x, t) \mapsto t^{\frac{1}{\alpha}} x$ we could even assume that $\alpha=1$, but we choose not to do so in order that the notation $t x$ retains its usual meaning when $\mathbb{X}$ is a subset of some vector space over $\mathbb{R}$.

We begin with a technical lemma.

Lemma 2.5. Suppose that $\nu$ is an $\alpha$-homogeneous measure on $(\mathbb{X}, \mathfrak{B})$. Write $\mathfrak{B}^{\nu}$ for the completion of the $\sigma$-algebra $\mathfrak{B}$ with respect to the measure $\nu$. Then $s B \in \mathfrak{B}^{\nu}$ with $\nu(s B)=s^{-\alpha} \nu(B)$ for all $s>0$ and $B \in \mathfrak{B}^{\nu}$.

Proof. Suppose that $A \subset \mathbb{X}$ is a $\nu$-null set. That is, there exists $N \in \mathfrak{B}$ such that $A \subseteq N$ and $\nu(N)=0$. For any $s>0, s A \subseteq s N \in \mathfrak{B}$ and $\nu(s N)=s^{-\alpha} \nu(N)=0$ by (2.1), and so $s A$ is also $\nu$-null. Now, $B \in \mathfrak{B}^{\nu}$ if and only if there exists $C \in \mathfrak{B}$ such that $B \triangle C$ is $\nu$-null, in which case $\nu(B)=\nu(C)$. Since $(s B) \triangle(s C)=s(B \triangle C)$ for $s>0$ and the latter set is $\nu$-null by the above, we see that $s B \in \mathfrak{B}^{\nu}$ and $\nu(s B)=\nu(s C)=s^{-\alpha} \nu(C)=s^{-\alpha} \nu(B)$.

Remark 2.6. Note that Lemma 2.5 implies that the map $x \mapsto s x$ is $\mathfrak{B}^{\nu} / \mathfrak{B}^{\nu}$-measurable for any $s>0$. It does not say that the map $(x, s) \mapsto$ $s x$ is $\mathfrak{B}^{\nu} \otimes \mathcal{B}\left(\mathbb{R}_{++}\right) / \mathfrak{B}^{\nu}$-measurable.

Notation 2.7. For $I \subseteq \mathbb{R}_{++}$and $B \in \mathfrak{B}$, put

$$
I B:=\bigcup_{t \in I} t B
$$

The following is the first of a series of related assumptions that require the existence of a suitably rich family of subsets or nonnegative functions on our carrier space.

Assumption A. There exist sets $U_{k} \in \mathfrak{B}, k \in \mathbb{N}$, such that

(i) if $x \in U_{k}$, then $t x \in U_{k}$ for all $t \geqslant 1$ (that is, $[1, \infty) U_{k}=U_{k}$ );

(ii) the sets $V_{k}:=(0, \infty) U_{k}, k \in \mathbb{N}$, cover $\mathbb{X}$ (that is, $\left.\bigcup_{k \in \mathbb{N}} V_{k}=\mathbb{X}\right)$.

Proposition 2.8. Suppose that Assumption A holds. The following are equivalent for a measure $\nu$ on $(\mathbb{X}, \mathfrak{B})$.

i) The measure $\nu$ is a nontrivial $\alpha$-homogeneous measure with

$$
\nu\left(U_{k}\right)<\infty, \quad k \in \mathbb{N} .
$$

ii) The measure $\nu$ is the push-forward of the measure $\pi \otimes \theta_{\alpha}$ by the map $(x, t) \mapsto t x$, where $\pi$ is a probability measure on $\mathbb{X}$ such that

$$
\int_{0}^{\infty} \pi\left(t U_{k}\right) t^{\alpha-1} d t<\infty, \quad k \in \mathbb{N},
$$

and $\theta_{\alpha}$ is the measure on $\mathbb{R}_{++}$given by $\theta_{\alpha}(d t):=\alpha t^{-(\alpha+1)} d t$. 
(iii) For a probability measure $\pi$ on $\mathbb{X}$ such that (2.3) holds,

$$
\nu(B)=\alpha \int_{0}^{\infty} \pi(t B) t^{\alpha-1} d t
$$

for all $B \in \mathfrak{B}$.

Proof. Statement (iii) is just a restatement of statement (ii), so it suffices to show that (i) $\Longrightarrow$ (ii) and (iii) $\Longrightarrow$ (i).

(i) $\Longrightarrow$ (ii). Fix $k \in \mathbb{N}$. Note that $V_{k}=\bigcup_{n \in \mathbb{N}} 2^{-n} U_{k}$, so that $V_{k} \in \mathfrak{B}$. Put

$$
\bar{U}_{k}:=\bigcap_{t<1} t U_{k}=\bigcap_{n \in \mathbb{N}}\left(1-2^{-n}\right) U_{k} \in \mathfrak{B},
$$

and observe that $V_{k}=(0, \infty) \bar{U}_{k}$. The $\alpha$-homogeneity of $\nu$ and $(2.2)$ yield that

$$
\nu\left(\bar{U}_{k}\right)=\inf _{t<1} t^{-\alpha} \nu\left(U_{k}\right)=\nu\left(U_{k}\right)<\infty
$$

For $x \in V_{k}$, set

$$
\tau(x):=\sup \left\{t>0: x \in t \bar{U}_{k}\right\} \in(0, \infty] .
$$

Since

$$
\left\{x \in V_{k}: \tau(x) \geqslant t\right\}=\bigcap_{s<t} s \bar{U}_{k}=t \bigcap_{s<1} s \bar{U}_{k}=t \bar{U}_{k},
$$

the function $\tau: V_{k} \mapsto(0, \infty]$ is $\mathfrak{B}$-measurable. Clearly, $\tau(s x)=s \tau(x)$ for all $s>0$. Also, setting

$$
N_{k}:=\left\{x \in V_{k}: \tau(x)=\infty\right\}=\bigcap_{t>0} t \bar{U}_{k},
$$

we have $s N_{k}=N_{k}$ for all $s>0$ and

$$
\nu\left(N_{k}\right)=\inf _{t>0} t^{-\alpha} \nu\left(\bar{U}_{k}\right)=0 .
$$

Define

$$
W_{k}:=\left\{x \in V_{k}: \tau(x)=1\right\}=\bar{U}_{k} \backslash\left(\bigcup_{t>1} t \bar{U}_{k}\right) \subseteq V_{k} \backslash N_{k} .
$$

Note that

$$
\bar{U}_{k} \backslash N_{k}=[1, \infty) W_{k}
$$

and

$$
V_{k} \backslash N_{k}=(0, \infty) W_{k} .
$$

Set $V_{1}^{\prime}:=V_{1}$ and $V_{i}^{\prime}:=\left(V_{i} \cap\left(V_{1} \cup \cdots \cup V_{i-1}\right)^{c}\right)$ for $i \geqslant 2$. Then put $V_{j}^{\prime \prime}=V_{j}^{\prime} \backslash N_{j}, W_{j}^{\prime \prime}:=W_{j} \cap V_{j}^{\prime \prime}$ and $\bar{U}_{j}^{\prime \prime}:=\bar{U}_{j} \cap V_{j}^{\prime \prime}$ for $j \in \mathbb{N}$. The sets $\left\{V_{k}^{\prime \prime}\right\}_{k \in \mathbb{N}}$ are disjoint, $\mathbb{X} \backslash \bigcup_{k \in \mathbb{N}} V_{k}^{\prime \prime} \subseteq \bigcup_{k \in \mathbb{N}} N_{k}$ is a $\nu$-null set, and $\bar{U}_{k}^{\prime \prime}=\left\{t x: t \geqslant 1, x \in W_{k}^{\prime \prime}\right\}$ for all $k \in \mathbb{N}$. Therefore, it is possible to 
assume without loss of generality that all of the sets $N_{k}$ are empty, the sets $\left\{\bar{U}_{k}\right\}_{k \in \mathbb{N}}$ are pairwise disjoint, the sets $\left\{V_{k}\right\}_{k \in \mathbb{N}}$ form a measurable partition of $\mathbb{X}$, and that $\tau(x)$ is uniquely defined and finite for all $x \in \mathbb{X}$.

The sets $\left\{x \in V_{k}: \tau(x)=t\right\}=t W_{k}$ are disjoint for different $t>0$ and their union is $V_{k}$. The map $x \mapsto\left(\tau(x)^{-1} x, \tau(x)\right)$ is therefore a well-defined bimeasurable bijection between $V_{k}$ and $W_{k} \times \mathbb{R}_{++}$. Let $\tilde{\nu}$ be the push-forward of $\nu$ by the map $x \mapsto\left(\tau(x)^{-1} x, \tau(x)\right)$ and define a measure $\rho_{k}$ on $W_{k}$ by $\rho_{k}(A)=\tilde{\nu}(A \times[1, \infty))$. Note that $\rho_{k}$ is a finite measure, since

$$
\rho_{k}\left(W_{k}\right)=\tilde{\nu}\left(W_{k} \times[1, \infty)\right)=\nu\left(\bar{U}_{k}\right)<\infty
$$

by (2.2). The $\alpha$-homogeneity of $\nu$ is equivalent to the scaling property $\tilde{\nu}(A \times s B)=s^{-\alpha} \tilde{\nu}(A \times B)$ for $s>0$, measurable $A \subseteq W_{k}$, and Borel $B \subseteq \mathbb{R}_{++}$. Thus, if we let $\theta_{\alpha}$ be the measure on $\mathbb{R}_{++}$given by $\theta_{\alpha}(d t)=$ $\alpha t^{-(\alpha+1)} d t$, then

$$
\begin{aligned}
\tilde{\nu}(A \times[b, \infty)) & =\tilde{\nu}(A \times b[1, \infty)) \\
& =b^{-\alpha} \tilde{\nu}(A \times[1, \infty)) \\
& =\rho_{k}(A) \times \theta_{\alpha}([b, \infty))
\end{aligned}
$$

for $A \subseteq W_{k}$. The restriction of $\tilde{\nu}$ to $W_{k} \times \mathbb{R}_{++}$is therefore $\rho_{k} \otimes \theta_{\alpha}$ and hence the restriction of $\nu$ to $V_{k}$ is the push-forward of $\rho_{k} \otimes \theta_{\alpha}$ by the map $(y, t) \mapsto t y$.

We can think of $\rho_{k}$ as a measure on all of $V_{k}$. For $c_{k} \in \mathbb{R}_{++}$, let $\eta_{k}$ be the measure on $V_{k}$ that assigns all of its mass to the set $c_{k} W_{k}$ and is given by $\eta_{k}(A)=c_{k}^{\alpha} \rho_{k}\left(c_{k}^{-1} A\right)$. We have

$$
\begin{aligned}
\left(\eta_{k} \otimes \theta_{\alpha}\right)(\{(y, t): t y \in B\}) & =\int \eta_{k}\left(t^{-1} B\right) \alpha t^{-(\alpha+1)} d t \\
& =\int c_{k}^{\alpha} \rho_{k}\left(c_{k}^{-1} t^{-1} B\right) \alpha t^{-(\alpha+1)} d t \\
& =\int \rho_{k}\left(s^{-1} B\right) \alpha s^{-(\alpha+1)} d s \\
& =\left(\rho_{k} \otimes \theta_{\alpha}\right)(\{(y, t): t y \in B\}) \\
& =\nu(B)
\end{aligned}
$$

for measurable $B \subset V_{k}$, and so $\eta_{k}$ is a finite measure with total mass $c_{k}^{\alpha} \rho_{k}\left(W_{k}\right)$ that has the property that the push-forward of $\eta_{k} \otimes \theta_{\alpha}$ by the map $(y, t) \mapsto t y$ is the restriction of $\nu$ to $V_{k}$.

We can regard $\eta_{k}$ as being a finite measure on all of $\mathbb{X}$ and, by choosing the constants $c_{k}, k \in \mathbb{N}$, appropriately we can arrange for $\pi:=\sum_{k \in \mathbb{N}} \eta_{k}$ to be a probability measure such that (2.4) holds, and this also implies (2.3). 
(iii) $\Longrightarrow($ i). It is easy to see that $\nu$ given by $(2.4)$ is $\alpha$-homogeneous, and (2.2) follows from (2.3) and (2.4).

Remark 2.9. A key observation in the proof of Proposition 2.8 is that it is possible to define measurable sets $W_{k}$ such that (2.5) and (2.6) hold, and if $x \in W_{k}$, then $t x \notin W_{k}$ for any $t \neq 1$. We would like to reverse the construction in Proposition 2.8 by starting with a suitable collection of sets $\left\{W_{k}\right\}_{k \in \mathbb{N}}$ with this last property and conclude that if we put $\bar{U}_{k}:=[1, \infty) W_{k}$, then $\bar{U}_{k} \in \mathfrak{B}$ and if we construct sets from each of the $\bar{U}_{k}$ in the manner that the $W_{k}$ are constructed in the proof, then we recover the $W_{k}$. There is, however, a slightly delicate point here: if $B \in \mathfrak{B}$, then it is not necessarily the case that $[1, \infty) B=\bigcup_{t \geqslant 1} t B=$ $\{t x: t \geqslant 1, x \in B\} \in \mathfrak{B}$.

Lemma 2.10. Let $\nu$ be a $\sigma$-finite $\alpha$-homogeneous measure on $(\mathbb{X}, \mathfrak{B})$ and suppose that $\mathfrak{B}$ is $\nu$-complete.

i) For $B \in \mathfrak{B}$ and a Borel set $I \subseteq \mathbb{R}_{++}$, the set $I B$ also belongs to $\mathfrak{B}$.

ii) If $\nu([t, u) B)<\infty$ for $B \in \mathfrak{B}$ and $0<t<u<\infty$, then $\nu([s, \infty) B)<\infty$ for all $s>0$.

Proof. For part (i), observe that

$$
\begin{aligned}
I B & =\{y \in \mathbb{X}: y=t x, \text { for some } t \in I \text { and } x \in B\} \\
& =\left\{y \in \mathbb{X}: t^{-1} y=x, \text { for some } t \in I \text { and } x \in B\right\} \\
& =\left\{y \in \mathbb{X}: u y=x, \text { for some } u \in I^{-1} \text { and } x \in B\right\} \\
& =\left\{y \in \mathbb{X}: u y \in B, \text { for some } u \in I^{-1}\right\} \\
& =\Pi\left(\left\{(y, u) \in \mathbb{X} \times I^{-1}: u y \in B\right\}\right),
\end{aligned}
$$

where $\Pi: \mathbb{X} \times \mathbb{R}_{++} \rightarrow \mathbb{X}$ is the projection map defined by $\Pi((x, t))=x$. The map $t \mapsto t^{-1}$ from $\mathbb{R}_{++}$to itself is a $\mathcal{B}\left(\mathbb{R}_{++}\right)$-measurable bijection and it is its own inverse, so the set $I^{-1}$ is $\mathcal{B}\left(\mathbb{R}_{++}\right)$-measurable. It follows that the set $\left\{(y, u) \in \mathbb{X} \times I^{-1}: u y \in B\right\}$ is $\mathfrak{B} \otimes \mathcal{B}\left(\mathbb{R}_{++}\right)$measurable. The projection theorem (see, for example, [14, Th. 12.3.4] or [7, Section III.44]) and the $\nu$-completeness of $\mathfrak{B}$ yield that $I B \in \mathfrak{B}$.

For part (ii), note that

$$
\begin{aligned}
{[s, \infty) B } & =\left(\bigcup_{n \geqslant 0} \frac{s}{t}\left(\frac{u}{t}\right)^{n}[t, u)\right) B \\
& \subseteq \bigcup_{n \geqslant 0} \frac{s}{t}\left(\frac{u}{t}\right)^{n}[t, u) B .
\end{aligned}
$$


By the $\alpha$-homogeneity of $\nu$

$$
\nu\left(\frac{s}{t}\left(\frac{u}{t}\right)^{n}[t, u) B\right)=\left(\frac{s}{t}\right)^{-\alpha}\left(\frac{u}{t}\right)^{-n \alpha} \nu([t, u) B),
$$

and the result follows from the subadditivity of $\nu$ and the summability of the relevant geometric series.

It is possible to obtain the conclusion of Proposition 2.8 under another assumption.

Assumption B. There exists $\mathbb{S} \in \mathfrak{B}$ such that

$$
\mathbb{X}=\left\{t x: t \in \mathbb{R}_{++}, x \in \mathbb{S}\right\},
$$

and $\{x, t x\} \subset \mathbb{S}$ for some $x \in \mathbb{X}$ and $t>0$ implies that $t=1$.

Definition 2.11. The orbits of the action of the group $\mathbb{R}_{++}$on $\mathbb{X}$ are the sets of the form $\left\{t x: t \in \mathbb{R}_{++}\right\}$for some $x \in \mathbb{X}$. The orbits are the equivalence classes for the equivalence relation on $\mathbb{X}$ where we declare $x$ and $y$ to be equivalent if $y=t x$ for some $t \in \mathbb{R}_{++}$. Assumption B says that the measurable set $\mathbb{S}$ intersects each equivalence class in exactly one point. Such a set is called a transversal.

The following result is immediate from Lemma 2.10 and the proof of Proposition 2.8.

Proposition 2.12. Let $\nu$ be a $\sigma$-finite, $\alpha$-homogeneous measure on $\mathbb{X}$.

i) Suppose that Assumption $A$ and the finiteness condition (2.2) hold. Set

$$
W_{k}:=\left(\bigcap_{s<1} s U_{k}\right) \backslash\left(\bigcup_{t>1} t\left(\bigcap_{s<1} s U_{k}\right)\right) .
$$

Then, by possibly replacing $\mathbb{X}$ with a set $\mathbb{X}^{\prime} \in \mathfrak{B}$ such that $t \mathbb{X}^{\prime}=\mathbb{X}^{\prime}$ for all $t>0$ and $\nu\left(\mathbb{X} \backslash \mathbb{X}^{\prime}\right)=0$, Assumption $B$ holds for $\mathbb{S}:=\bigcup_{k \in \mathbb{N}} W_{k}$. Moreover, for any nonempty intervals $I_{k}:=\left[a_{k}, b_{k}\right) \subset \mathbb{R}_{++}, k \in \mathbb{N}$, it is the case that $I_{k} W_{k} \in \mathfrak{B}$ and $\nu\left(I_{k} W_{k}\right)<\infty$ for $k \in \mathbb{N}$.

ii) Suppose that that Assumption $B$ holds and there is a pairwise disjoint family of measurable sets $\left\{W_{k}\right\}_{k \in \mathbb{N}}$ such that $\mathbb{S}=$ $\bigcup_{k \in \mathbb{N}} W_{k}$ and a family of nonempty intervals $I_{k}:=\left[a_{k}, b_{k}\right) \subset$ $\mathbb{R}_{++}, k \in \mathbb{N}$, such that $\nu\left(I_{k} W_{k}\right)<\infty$ for all $k \in \mathbb{N}$, where $I_{k} W_{k}$ is guaranteed to belong to $\mathfrak{B}^{\nu}$ for all $k \in \mathbb{N}$. Then Assumption $A$ and the finiteness condition $(2.2)$ hold with $(\mathbb{X}, \mathfrak{B})$ replaced by $\left(\mathbb{X}, \mathfrak{B}^{\nu}\right)$ and $U_{k}:=[1, \infty) W_{k}$ for $k \in \mathbb{N}$. 
Definition 2.13. Recall that a Borel space is a measurable space that is Borel isomorphic to a Borel subset of a Polish space equipped with the trace of the Borel $\sigma$-algebra on the Polish space.

Lemma 2.14. Suppose that $\nu$ is a $\sigma$-finite $\alpha$-homogeneous measure on $(\mathbb{X}, \mathfrak{B})$ and that Assumption $B$ is satisfied. For $x \in \mathbb{X}$, define $\tau(x) \in$ $\mathbb{R}_{++}$by the requirement that $\tau(x)^{-1} x \in \mathbb{S}$. Then the following hold.

i) The maps $(x, t) \mapsto$ tx from $\mathbb{S} \times \mathbb{R}_{++}$to $\mathbb{X}$ and $x \mapsto$ $\left(\tau(x)^{-1} x, \tau(x)\right)$ from $\mathbb{X}$ to $\mathbb{S} \times \mathbb{R}_{++}$are mutually inverse.

ii) If $\mathfrak{B}$ is $\nu$-complete, then $x \mapsto\left(\tau(x)^{-1} x, \tau(x)\right)$ is $\mathfrak{B} / \mathfrak{B}_{\mid \mathbb{S}} \otimes$ $\mathcal{B}\left(\mathbb{R}_{++}\right)$-measurable, where $\mathfrak{B}_{\mid \mathbb{S}}$ is the $\sigma$-algebra induced by $\mathfrak{B}$ on $\mathbb{S}$.

iii) If $(\mathbb{X}, \mathfrak{B})$ is a Borel space, then $x \mapsto\left(\tau(x)^{-1} x, \tau(x)\right)$ is $\mathfrak{B} / \mathfrak{B}_{\mathbb{S}} \otimes$ $\mathcal{B}\left(\mathbb{R}_{++}\right)$-measurable.

Proof. Part (i) is clear from the definition of $\tau$. Turning to part (ii), it suffices to show that the map $\tau$ is $\mathfrak{B}$-measurable, but this follows from Lemma 2.10 and the fact that $\{x \in \mathbb{X}: \tau(x) \geqslant t\}=[t, \infty) \mathbb{S}$. For part (iii), first note that if $\mathbb{X}$ is a Borel space, then so is $\mathbb{S}$ equipped with the trace $\sigma$-algebra and hence also $\mathbb{S} \times \mathbb{R}_{++}$. Now apply the result of Kuratowski (see, for example, [22, Section I.3]) that a measurable bijection between two Borel spaces has a measurable inverse.

Proposition 2.8 required Assumption A concerning the existence of suitable countable family of subsets of the carrier space $\mathbb{X}$ and the hypothesis (2.2) that these sets all have finite $\nu$ mass. The following result replaces such requirements by the finiteness of integrals $\int h d \nu$ for a countable family of measurable functions $h: \mathbb{X} \mapsto \mathbb{R}_{+}, n \in \mathbb{N}$.

Theorem 2.15. Suppose that Assumption B holds. Let $\mathcal{H}$ be a countable family of measurable functions $h: \mathbb{X} \mapsto \mathbb{R}_{+}$such that for all $h \in \mathcal{H}$ and $x \in \mathbb{X}$ the function $t \mapsto h(t x)$ is right-continuous (or leftcontinuous) on $\mathbb{R}_{++}$.

$A \sigma$-finite measure $\nu$ on $\mathbb{X}$ is $\alpha$-homogeneous and satisfies

$$
\int_{\mathbb{X}} h(x) \nu(d x)<\infty, \quad h \in \mathcal{H}
$$

and

$$
\nu\left(\bigcap_{h \in \mathcal{H}}\{x \in \mathbb{X}: h(x)=0\}\right)=0
$$


if and only if there exists a probability measure $\pi$ on $\mathbb{X}$ such that (2.4) holds,

$$
\int_{\mathbb{X}} \int_{0}^{\infty} h(t x) t^{-(\alpha+1)} d t \pi(d x)<\infty, \quad h \in \mathcal{H}
$$

and

$$
\pi\left((0, \infty) \bigcap_{h \in \mathcal{H}}\{x \in \mathbb{X}: h(x)=0\}\right)=0 .
$$

Proof. Necessity. Enumerate $\mathcal{H}$ as $\left\{h_{n}\right\}_{n \in \mathbb{N}}$. Assume that for all $x \in \mathbb{X}$ the function $t \mapsto h_{n}(t x)$ is right-continuous in $t \in \mathbb{R}_{++}$. Denote by $\mathbb{Q}_{++}$the set of strictly positive rational numbers. For $n, k, j \in \mathbb{N}$ and $r \in \mathbb{Q}_{++}$, define

$$
U_{n k r j}:=\left\{x \in \mathbb{S}: h_{n}(s x) \geqslant 2^{-k}, s \in\left[r, r+2^{-j}\right]\right\} .
$$

Since $B:=\left\{x \in \mathbb{S}: h_{n}(x) \geqslant 2^{-k}\right\} \in \mathfrak{B}$, the right-continuity property and Remark 2.2 yield that

$$
U_{n k r j}=\bigcap_{s \in \mathbb{Q} \cap\left[r, r+2^{-j}\right]} s^{-1} B \in \mathfrak{B} .
$$

Put $J_{r j}:=\left[r, r+2^{-j}\right]$. Then $J_{n j} U_{n k r j} \in \mathfrak{B}^{\nu}$ and, by (2.7),

$$
\nu\left(J_{n j} U_{n k r j}\right) \leqslant 2^{k} \int_{J_{n j} U_{n k r j}} h_{n}(x) \nu(d x) \leqslant 2^{k} \int_{\mathbb{X}} h_{n}(x) \nu(d x)<\infty .
$$

Put $\mathbb{S}^{\prime}=\bigcup_{n, k, r, j} U_{n k r j}$. If $x \in \mathbb{S} \backslash \mathbb{S}^{\prime}, n, k, j \in \mathbb{N}$, and $r \in \mathbb{Q}_{++}$, there exists $s \in\left[r, r+2^{-j}\right]$ (depending on $\left.x, n, k, r, j\right)$ such that $h_{n}(s x)<$ $2^{-k}$. The right-continuity of $t \mapsto h_{n}(t x)$ yields that $h_{n}(r x)=0$ for all $r \in \mathbb{Q}_{++}$and thence $h_{n}(t x)=0$ for all $t \in \mathbb{R}_{++}$.

Enumerate $\left(U_{n k r j}, J_{r j}\right), n, k, j \in \mathbb{N}$ and $r \in \mathbb{Q}_{++}$, as $\left(W_{n}, I_{n}\right), n \in \mathbb{N}$, so that $\mathbb{S}^{\prime}=\bigcup_{n \in \mathbb{N}} W_{n}$ and $\nu\left(I_{n} W_{n}\right)<\infty$ for all $n \in \mathbb{N}$. By (2.8), the measure $\nu$ assigns all of its mass to the set $\mathbb{X}^{\prime}:=(0, \infty) \mathbb{S}^{\prime} \in \mathfrak{B}^{\nu}$, and the result follows from the decomposition of $\nu$ guaranteed by Proposition 2.8 and Proposition 2.12. Finally, (2.9) follows from the change of variables in (2.7) using the polar decomposition of $\nu$ as $\pi \otimes \theta_{\alpha}$.

If all functions $h_{n}(t x)$ are left continuous in $t \in \mathbb{R}_{++}$, then the definition of $U_{n k r j}$ should be modified by working with the interval $\left[r-2^{-j}, r\right]$ for $2^{-j}<r$.

Sufficiency is immediate by checking that the measure $\nu$ defined by (2.4) is $\alpha$-homogeneous.

Remark 2.16. If $h_{n}(t x) \rightarrow 0$ as $t \downarrow 0$ for all $n \in \mathbb{N}$, then it is not necessary to require Assumption $\mathrm{B}$ in Theorem 2.15. A measurable 
transversal can be constructed as follows. For each $n \in \mathbb{N}$, let

$$
W_{n}:=\left\{x \in \mathbb{X}: h_{k}(x)=0, k<n, h_{n}(x) \neq 0\right\} .
$$

Next, partition $W_{n}$ into measurable sets

$$
\begin{aligned}
& W_{n j}:=\left\{x \in W_{n}: \sup _{t>0} h_{n}(t x) \in\left(2^{-j}, 2^{-j+1}\right]\right\}, \quad j \geqslant 1, \\
& W_{n 0}:=\left\{x \in W_{n}: \sup _{t>0} h_{n}(t x)>1\right\} .
\end{aligned}
$$

Finally, define

$$
\tau_{n j}(x):=\inf \left\{t>0: h_{n}(t x)>2^{-j}\right\}, \quad x \in W_{n j},
$$

and

$$
S_{n j}:=\left\{\tau_{n j}(x)^{-1} x: x \in W_{n j}\right\} .
$$

Then $\mathbb{S}:=\bigcup_{n, j \in \mathbb{N}} S_{n j}$ satisfies Assumption $\mathbb{B}$ in the complement of the set $\left\{x \in \mathbb{X}: h_{n}(x)=0, n \in \mathbb{N}\right\}$.

Remark 2.17. Theorem 2.15 asserts that an $\alpha$-homogeneous measure $\nu$ is the push-forward of the measure $\pi \otimes \theta_{\alpha}$ under the map $(x, t) \mapsto$ tx from $\mathbb{X} \times \mathbb{R}_{++}$to $\mathbb{X}$. In this case we say that $\nu$ admits a polar representation. It follows from the proof that we may replace $\mathbb{X}$ by a subset that is invariant under the action of $\mathbb{R}_{++}$in such a way that the probability measure $\pi$ assigns all of its mass to a transversal.

\section{Strictly Stable RANDOM Elements on CONVEX CONES}

Definition 3.1. A convex cone $\mathbb{K}$ is an abelian measurable semigroup with neutral element $\mathbf{e}$ and a scaling $(x, a) \mapsto a x$ by $\mathbb{R}_{++}$that has the properties

$$
\begin{aligned}
a(x+y) & =a x+a y, \quad a>0, x, y \in \mathbb{K}, \\
a \mathbf{e} & =\mathbf{e}, \quad a>0 .
\end{aligned}
$$

Remark 3.2. The simplest examples of convex cones are $\mathbb{K}=\mathbb{R}^{d}$ and $\mathbb{K}=\mathbb{R}_{+}^{d}$ with the usual scaling by $\mathbb{R}_{++}$, but there are many other examples, some of which we will recall later in this paper.

Remark 3.3. In contrast to the many classical studies of convex cones that are convex subsets of vector spaces over the reals which are closed under multiplication by nonnegative scalars (see, for example, [10]), we do not assume the validity of the second distributivity law; that is, we do not require that $a x+b x=(a+b) x$ for $a, b>0$ and $x \in \mathbb{K}$.

Stable random elements of convex cones have been studied in [5] under the assumptions that the scaling is jointly continuous and that $\mathbb{K}^{\prime}:=\mathbb{K} \backslash\{\mathbf{e}\}$ is a Polish space. 
Definition 3.4. An involution is a measurable map $x \mapsto x^{*}$ satisfying $(x+y)^{*}=x^{*}+y^{*},(a x)^{*}=a x^{*}$, and $\left(x^{*}\right)^{*}=x$ for all $x, y \in \mathbb{K}$ and $a>0$. We assume that $\mathbb{K}$ is equipped with an involution.

Definition 3.5. A measurable function $\chi$ that maps $\mathbb{K}$ into the unit disk $\mathbb{D}$ in the complex plane is called a bounded semicharacter (or, more briefly, a character $)$ if $\chi(\mathbf{e})=1, \chi(x+y)=\chi(x) \chi(y)$, and $\chi\left(x^{*}\right)=\overline{\chi(x)}$ for all $x, y \in \mathbb{K}$.

Remark 3.6. The family of all characters form a convex cone when equipped with pointwise multiplication as the semigroup operation, the topology of pointwise convergence, the neutral element $\mathbf{1}$ being the character identically equal to 1 , the involution being the complex conjugate, and the scaling defined by $(a \chi)(x):=\chi(a x), x \in \mathbb{K}, a>0$.

We assume in the following that there exists a separating family $\hat{\mathbb{K}}$ of characters in the usual sense that for each $x \neq y$ there exists $\chi \in \hat{\mathbb{K}}$ such that $\chi(x) \neq \chi(y)$. Such a family does not exist for all semigroups, see [5, Ex. 8.20]. We also assume that the characters in $\hat{\mathbb{K}}$ generate the $\sigma$-algebra on $\mathbb{K}$ and that the family $\hat{\mathbb{K}}$ is closed under taking finite products, contains the constant function 1 , and so is a semigroup itself. Note that $\hat{\mathbb{K}}$ is not assumed to be closed under scaling and so it is not necessarily a convex cone.

The distribution of a $\mathbb{K}$-valued random element $\xi$ is determined by its Laplace transform $\mathbb{E} \chi(\xi), \chi \in \hat{\mathbb{K}}$, (see, for example, [5, Th. 5.4]). A random element $\xi$ is said to be symmetric if $\xi \stackrel{d}{\sim} \xi^{*}$, that is, $\xi$ coincides in distribution with its involution. The Laplace transform of a symmetric random element takes only real values. Recall that the classical LévyKhinchin-Itô description of infinitely divisible random elements of $\mathbb{R}^{d}$ can involve subtracting "compensating" terms to achieve convergence of a sum of the points in a Poisson point process that would otherwise be divergent, but that such compensation is not necessary when the random elements are symmetric in the usual sense for $\mathbb{R}^{d}$-valued random elements (which is the special case of the sense considered here with the involution given by $x \mapsto-x)$. Since no such recentering using subtraction is possible in the general semigroup setting, we mostly consider symmetric random elements. If the involution is the identity, then all random elements are symmetric.

Definition 3.7. A random element $\xi$ in $\mathbb{K}$ is said to be strictly $\alpha$-stable if

$$
a^{1 / \alpha} \xi^{\prime}+b^{1 / \alpha} \xi^{\prime \prime} \stackrel{d}{\sim}(a+b)^{1 / \alpha} \xi, \quad a, b>0,
$$

where $\xi^{\prime}$ and $\xi^{\prime \prime}$ are i.i.d. copies of $\xi$. 
In general cones, any value $\alpha \neq 0$ is possible, see [5]. Since by redefining the scaling from $a x$ to $a^{-1} x$ it is possible to turn a negative $\alpha$ into a positive one, in the following we consider only the case $\alpha>0$.

Remark 3.8. An alternative definition of strictly stable random elements that coincides with the above for $\mathbb{R}^{d}$ (and in many other situations) requires that, for all $n \geqslant 2$, there exists $a_{n}>0$ such that $\xi_{1}+\cdots+\xi_{n} \stackrel{d}{\sim} a_{n} \xi$, where $\xi, \xi_{1}, \ldots, \xi_{n}$ are i.i.d. and $n \geqslant 2$. While (3.1) implies this condition immediately, extra assumptions related to the semicontinuity property of characters and the continuity of the scaling operation are needed for the equivalence of the two definitions, see [5, Th. 5.16]. The major step is to establish that $a_{n}=n^{1 / \alpha}$, after which (3.1) follows easily.

A strictly $\alpha$-stable $\xi$ is always infinitely divisible and so its Laplace transform satisfies

$$
\mathbb{E} \chi(\xi)=\exp \{-\varphi(\chi)\}, \quad \chi \in \hat{\mathbb{K}},
$$

for a negative definite complex-valued function $\varphi$ on $\hat{\mathbb{K}}$ with $\operatorname{Re} \varphi \in$ $[0, \infty]$ and $\varphi(\mathbf{1})=0$, see [3, Th. 3.2.2, Prop. 4.3.1]. The function $\varphi$ is called the Laplace exponent of $\xi$.

A Laplace exponent is associated with a unique Lévy measure $\nu$, a Radon measure on the semigroup of all bounded characters on $\hat{\mathbb{K}}$, see [3]. In the following we always assume that the Lévy measure is supported by $\mathbb{K}^{\prime}:=\mathbb{K} \backslash\{\mathbf{e}\}$ canonically embedded into the semigroup of all bounded semicharacters on $\hat{\mathbb{K}}$ and that it is $\sigma$-finite on $\mathbb{K}^{\prime}$. In this case we say that $\xi$ admits a Lévy measure. The Lévy measure $\nu$ satisfies

$$
\int_{\mathbb{K}^{\prime}}(1-\operatorname{Re} \chi(x)) \nu(d x)<\infty
$$

for all $\chi \in \hat{\mathbb{K}}$. The measure $\nu$ is the intensity measure of a Poisson process $\left\{\eta_{i}: i \in \mathbb{N}\right\}$ on $\mathbb{K}^{\prime}$, and the appropriately defined (if necessary using principal values and compensating terms) sum of the points $\eta_{i}$ yields an infinitely divisible random element that is said to not have deterministic, Gaussian or idempotent components.

Lemma 3.9. Assume for some $\chi \in \hat{\mathbb{K}}$ that

$$
\liminf _{t \downarrow 0} \operatorname{Re} \chi(t x)>0
$$

for all $x \in \mathbb{K}$. If $\xi$ is a strictly $\alpha$-stable random element, then $\mathbb{E} \chi(\xi) \neq$ 0 . 
Proof. Since $-1 \leqslant \operatorname{Re} \chi(t x) \leqslant 1$ for all $t>0$ and $x \in \mathbb{K}$, it follows from the assumption (3.4) and Fatou's Lemma that

$$
0<\mathbb{E}\left[\liminf _{t \downarrow 0} \operatorname{Re} \chi(t \xi)\right] \leqslant \liminf _{t \downarrow 0} \mathbb{E R e} \chi(t \xi) .
$$

By the stability property of $\xi, \mathbb{E} \chi(\xi)=\left(\mathbb{E} \chi\left(n^{-1 / \alpha} \xi\right)\right)^{n}$ for all $n \in \mathbb{N}$, and so $\mathbb{E} \chi(\xi)=0$ would imply that $\mathbb{E} \chi\left(n^{-1 / \alpha} \xi\right)=0$ and hence $\mathbb{E} \operatorname{Re} \chi\left(n^{-1 / \alpha} \xi\right)=0$ for all $n \in \mathbb{N}$, but this contradicts (3.5).

Lemma 3.10. Assume that (3.4) holds for all $\chi \in \hat{\mathbb{K}}$. Then the Lévy measure of a strictly $\alpha$-stable random element is an $\alpha$-homogeneous measure on $\mathbb{K}^{\prime}$.

Proof. It follows from (3.1) that

$$
\varphi\left(a^{1 / \alpha} \chi\right)+\varphi\left(b^{1 / \alpha} \chi\right)=\varphi\left((a+b)^{1 / \alpha} \chi\right), \quad a, b>0,
$$

where the Laplace exponent $\varphi$ of the strictly stable random element $\xi$ is finite by Lemma 3.9. Since $(x, a) \mapsto a x$ is a jointly measurable map, the function $a \mapsto \mathbb{E} \chi(a \xi)$ is measurable by Fubini's theorem. Therefore,

$$
\varphi(a \chi)=a^{\alpha} \varphi(\chi), \quad a>0 .
$$

The random element $a \xi$ is also infinitely divisible and its Lévy measure is $B \mapsto \nu(a B)$ for measurable subsets $B \subseteq \mathbb{K}^{\prime}$. By (3.6), the Lévy measure of $a \xi$ is $a^{\alpha} \nu$. Since the Lévy measure is unique, we obtain (2.1).

Theorem 3.11. Let $\xi$ be a strictly stable random element that admits a $\sigma$-finite Lévy measure $\nu$. Assume that there is a countable, closed under finite products, separating family of characters $\hat{\mathbb{K}}$ such that $t \mapsto$ $\operatorname{Re} \chi(t x), t \in \mathbb{R}_{++}$, is right- (or left-) continuous for all $x \in \mathbb{K}^{\prime}$ and $\chi \in \hat{\mathbb{K}}$. Assume also that Assumption B holds and (3.4) holds for all $\chi \in \hat{\mathbb{K}}$.

Then $\nu$ admits the polar representation $\pi \otimes \theta_{\alpha}$, where $\pi$ is a probability measure on $\mathbb{K}^{\prime}$ satisfying

$$
\int_{0}^{\infty} \mathbb{E}[1-\operatorname{Re} \chi(t \varepsilon)] t^{-(\alpha+1)} d t<\infty, \quad \chi \in \hat{\mathbb{K}} .
$$

The Poisson process with intensity measure $\nu$ can be represented as $\left\{\Gamma_{i}^{-1 / \alpha} \varepsilon_{i}\right\}_{i \in \mathbb{N}}$, where $\left\{\varepsilon_{i}\right\}_{i \in \mathbb{N}}$ is a sequence of $i . i . d$. copies of a random element $\varepsilon$ in $\mathbb{K}^{\prime}$ with distribution $\pi$, and $\left\{\Gamma_{i}\right\}_{i \in \mathbb{N}}$ are successive points of a unit intensity Poisson process on $\mathbb{R}_{+}$. If $\xi$ is symmetric, then $\varepsilon$ can also be chosen to have a symmetric distribution. 
Proof. The measure $\nu$ admits the polar decomposition $\pi \otimes \theta_{\alpha}$ by Theorem 2.15 applied with $\mathbb{X}:=\mathbb{K}^{\prime}, \mathcal{H}:=\{1-\operatorname{Re} \chi: \quad \chi \in \hat{\mathbb{K}}\}$, and $\nu$ being the Lévy measure of $\xi$. Indeed, (2.7) follows from (3.3), $\nu$ is $\alpha$ homogeneous by Lemma 3.10 given that $(3.4)$ is assumed to hold, and the separating condition imposed on $\hat{\mathbb{K}}$ yields (2.8).

The Poisson point process with intensity measure $\theta_{\alpha}$ is obtained as $\left\{\Gamma_{i}^{-1 / \alpha}: i \in \mathbb{N}\right\}$. Thus, the Poisson process on $\mathbb{K}^{\prime} \times \mathbb{R}_{++}$with intensity measure $\pi \otimes \theta_{\alpha}$ is obtained by marking a Poisson point process $\left\{\Gamma_{i}^{-1 / \alpha}\right.$ : $i \in \mathbb{N}\}$ with marks $\left\{\varepsilon_{i}\right\}_{i \in \mathbb{N}}$ that are i.i.d. copies of a random element $\varepsilon$ in $\mathbb{K}^{\prime}$ with distribution $\pi$. By (2.9), the random element $\varepsilon$ satisfies (3.7). Since $\nu$ is the push-forward of $\pi \otimes \theta_{\alpha}$ under the multiplication map $(x, t) \mapsto t x$, the Poisson process with intensity $\nu$ is given by $\left\{\Gamma_{i}^{-1 / \alpha} \varepsilon_{i}\right.$ : $i \in \mathbb{N}\}$.

The uniqueness of the Lévy measure yields that $\nu$ is symmetric if $\xi$ is symmetric. Then in the proof of Proposition 2.8 it suffices to replace each set $W_{k}$ with the union of $W_{k}$ and its image under the involution to ensure that $\pi$ is a symmetric measure.

Remark 3.12. Note that the random element $\varepsilon$ in Theorem 3.11 is not unique and also is not restricted to belong to the transversal $\mathbb{S}$ from Assumption $\mathbb{B}$. By rescaling it is always possible to arrange that $\varepsilon \in \mathbb{S}$ a.s., however in this case, the Poisson process with intensity $\nu$ is given by $\left\{c \Gamma_{i}^{-1 / \alpha} \varepsilon_{i}: i \in \mathbb{N}\right\}$ for a positive scaling constant $c$.

Remark 3.13. Suppose that the "Lévy-Khinchin-Itô" decomposition of the strictly $\alpha$-stable random element $\xi$ does not contain any deterministic, Gaussian or idempotent components, so that $\xi$ is the sum of the points in a Poisson process $\left\{\eta_{i}: i \in \mathbb{N}\right\}$, where the sum is appropriately defined by using principal values and compensating terms if necessary, see [5, Th. 7.2]. Theorem 3.11 establishes that $\left\{\eta_{i}: i \in \mathbb{N}\right\}$ can be constructed as $\left\{\Gamma_{i}^{-1 / \alpha} \varepsilon_{i}: i \in \mathbb{N}\right\}$, that is, as randomly scaled i.i.d. copies of a random element $\varepsilon$.

Recall that no compensating terms for the sum of the $\eta_{i}$ are required if $\xi$ is $\alpha$-stable and symmetric. In this case, the Laplace exponent of $\xi$ is given by

$$
\varphi(\chi)=\int_{\mathbb{K}^{\prime}}(1-\chi(x)) \nu(d x)=\int_{\mathbb{K}^{\prime}}(1-\operatorname{Re} \chi(x)) \nu(d x), \quad \chi \in \hat{\mathbb{K}} .
$$

Put

$$
\xi^{(r)}:=\sum_{\Gamma_{i} \leqslant r} \Gamma_{i}^{-1 / \alpha} \varepsilon_{i}, \quad r>0
$$


The probability generating functional formula for a Poisson point process yields that, for all $\chi \in \hat{\mathbb{K}}$,

$$
\begin{aligned}
\mathbb{E} \chi\left(\xi^{(r)}\right) & =\exp \left\{-\int_{r^{-\alpha}}^{\infty} \int_{\mathbb{K}^{\prime}}(1-\chi(t x)) \alpha t^{-(\alpha+1)} \pi(d x) d t\right\} \\
& \rightarrow \mathbb{E} \chi(\xi) \quad \text { as } r \uparrow \infty,
\end{aligned}
$$

since $\mathbb{E} \chi(t \varepsilon)$ is real-valued by the symmetry of $\varepsilon$ and the integral under the exponential converges by (3.7).

\section{EXAMPLES}

Example 4.1. If $\mathbb{K}=\mathbb{R}^{d}$ with the usual arithmetic addition, the involution given by the negation, the Borel $\sigma$-algebra, and conventional scaling by positive scalars, then Assumption $B$ holds with $\mathbb{S}$ being the sphere in $\mathbb{R}^{d}$ with respect to any norm. A countable separating family of continuous characters is given by $\chi(x)=\exp \{\imath\langle u, x\rangle\}$, where $\langle u, x\rangle$ is the scalar product of $u \in \mathbb{Q}^{d}$ and $x \in \mathbb{R}^{d}$, and Theorem 3.11 yields the polar representation of Lévy measures for strictly stable random vectors. In particular, each strictly stable random vector $\xi$ corresponds to the Poisson process $\left\{\Gamma_{i}^{-1 / \alpha} \varepsilon_{i}: i \in \mathbb{N}\right\}$. If $\alpha \in(0,1)$ or if $\xi$ is symmetric, then the sum of these points converges almost surely and yields the LePage series decomposition of $\xi$, see [16].

Example 4.2. In the setting of Example 4.1, define the scaling by letting $t x:=\exp \{(\log t) \mathbf{A}\} x, t>0$, for a non-degenerate matrix $\mathbf{A}$. The corresponding stable elements are usually called operator stable, see [12, 28]. An operator stable random element is infinitely divisible and so admits a series representation and a Lévy measure. By Theorem 3.11 , an operator stable random element $\xi$ in $\mathbb{R}^{d}$ admits the LePage series representation

$$
\xi \stackrel{d}{\sim} \sum_{i \in \mathbb{N}} \exp \left\{-\alpha^{-1}\left(\log \Gamma_{i}\right) \mathbf{A}\right\} \varepsilon_{i}
$$

Note that the polar decomposition of the Lévy measure appears in [11, Eq. (2)] and [12].

Example 4.3. Let $\mathbb{K}$ be the family $\mathbb{R}^{[0, \infty)}$ of real valued-functions on $\mathbb{R}_{+}$with the cylindrical $\sigma$-algebra, the pointwise arithmetic addition of functions as the semigroup operation, involution being the negation, and the scaling operation applied pointwise. It is known [13, 18] that an infinitely divisible separable in probability stochastic process $\xi(s)$, $s \in \mathbb{R}_{+}$, can be associated with the Poisson process $\left\{\eta_{i}, i \in \mathbb{N}\right\}$ on $\mathbb{R}^{[0, \infty)}$ with a $\sigma$-finite intensity measure $\nu$, so that $\xi$ admits a Lévy measure 
$\nu$. If $\xi$ is symmetric, then, for each $s \in \mathbb{R}_{+}$,

$$
\xi(s) \stackrel{d}{\sim} \lim _{r \downarrow 0} \sum_{\left|\eta_{i}(t)\right| \geqslant r} \eta_{i}(s), \quad s \in \mathbb{R}_{+}
$$

where $\stackrel{d}{\sim}$ in this setting denotes the equality of all finite-dimensional distributions. It should be noted that the order of summands in (4.1) may change with $t$, and there might be no order of the summands that guarantees the convergence for all rational $s$, not to say for all $s \in \mathbb{R}_{+}$. Such a common order exists for random functions with non-negative values (in which case $\mathbb{K}$ is endowed with the identical involution).

This cone does not admit a countable separating family of characters. Let $\tilde{\mathbb{K}}$ be the countable family of characters defined by

$$
\chi(x)=e^{\imath x(s) u}, \quad s \in \mathbb{Q}_{+},
$$

where $x=(x(s))_{s \geqslant 0}$ is an element of $\mathbb{R}^{[0, \infty)}$ and $u$ belongs to the set $\mathbb{Q}$ of rational numbers. Theorem 3.11 applies to the process $\xi$ restricted onto the set $\mathbb{Q}_{+}$of non-negative rationals, so that the image $\tilde{\nu}$ of the Lévy measure $\nu$ under the map that restricts a function to $\mathbb{Q}_{+}$has polar decomposition as $\pi \otimes \theta_{\alpha}$ and the Poisson process with intensity $\tilde{\nu}$ is given by $\left\{\Gamma_{i}^{-1 / \alpha} \tilde{\varepsilon}_{i}, i \geqslant 1\right\}$. By [13, Prop. 2.19], for each $t \in \mathbb{R}_{+} \backslash \mathbb{Q}_{+}$, the separability property of $\xi$ yields that $\Gamma_{i}^{-1 / \alpha} \tilde{\varepsilon}_{i}\left(t_{n}\right)$ converges in probability to $\eta_{i}(t)$, whence $\tilde{\varepsilon}_{i}\left(t_{n}\right)$ converges in probability to $\varepsilon_{i}(t):=\Gamma_{i}^{1 / \alpha} \eta_{i}(t)$. Thus, the Lévy measure $\nu$ corresponds to the Poisson process $\left\{\Gamma_{i}^{-1 / \alpha} \varepsilon_{i}, i \geqslant 1\right\}$, where $\left\{\varepsilon_{i}, i \in \mathbb{N}\right\}$ is a sequence of i.i.d. separable processes distributed as $\varepsilon(s), s \in \mathbb{R}_{+}$. Condition (3.7) in this setting is equivalent to $\mathbb{E}|\varepsilon(s)|^{\alpha}<\infty$ for all $s$.

In the symmetric case, following (3.8), the convergence in (4.1) can be rephrased as

$$
\xi(s) \stackrel{d}{\sim} \lim _{r \uparrow \infty} \sum_{\Gamma_{i} \leqslant r} \Gamma_{i}^{-1 / \alpha} \varepsilon_{i}(s), \quad s \in \mathbb{R}_{+} .
$$

Therefore, in case of symmetric $\alpha$-stable processes the order of summands in (4.1) can be made the same for all time points. This makes it possible to appeal to path regularity results for stochastic integrals from [24, Th. 4] in order to confirm that $\varepsilon$ shares path regularity properties with $\xi$, for instance, $\varepsilon$ is almost surely right-continuous with left limits (càdlàg) if $\xi$ is càdlàg. The same holds for stochastic processes with almost surely non-negative values, since then the involution is the identity. The pointwise convergence of the LePage series yields the uniform convergence, see [2]. Note that a result concerning the existence 
of the series representation of a general (not necessarily symmetric stable) infinitely divisible càdlàg function using càdlàg summands is not available.

Example 4.4. Let $\mathbb{K}$ be the family of non-negative functions $x(s)$, $s \in \mathbb{R}_{+}$, with the cylindrical $\sigma$-algebra, the semigroup operation being pointwise maximum, identical involution, and the scaling applied poinwisely to the values of the function. It is shown in [13] that each separable max-infinitely divisible stochastic process admits a Lévy measure. A separating family of characters is given by those of the form $x \mapsto \chi(x)=\mathbf{1}_{x(s)<a}$ for $s, a \in \mathbb{R}_{+}$. While these characters are not continuous, the function $t \mapsto \chi(t x)$ is right-continuous. Restricting the functions to non-negative rationals as in Example 4.3 and using the results from [13] concerning the max-infinitely divisible setting, we obtain that the Lévy measure of each max-stable separable in probability stochastic process $\xi$ admits a polar representation and the process itself admits the series representation

$$
\xi(s) \stackrel{d}{\sim} \bigvee_{i \in \mathbb{N}} \Gamma_{i}^{-1 / \alpha} \varepsilon_{i}(s),
$$

which first appeared in [6].

Example 4.5. Equip the family $\mathbb{K}$ of real-valued càdlàg functions on $\mathbb{R}_{+}$ that vanish at the origin with pointwise arithmetic addition, involution being the negation, and the scaling defined by $(t x)(s):=x(t s), s \in \mathbb{R}_{+}$, for $t \in \mathbb{R}_{++}$. Stable elements in this cone with $\alpha=1$ are called timestable processes in [15] and processes infinitely divisible with respect to time in [17]. The characters are given by (4.2) and, in view of the càdlàg assumption, they constitute a countable separating family and are right-continuous as required in Theorem 3.11. Furthermore, $\chi(t x)=\exp \{\imath x(t s) u\} \rightarrow 1$ as $t \downarrow 0$, so that a transversal in $\mathbb{K}$ can be constructed as in Remark 2.16, see also [15]. Thus, if a time-stable process with symmetric distribution admits a series representation with càdlàg functions, it also admits the LePage representation

$$
\xi(s) \stackrel{d}{\sim} \sum_{i \in \mathbb{N}} \varepsilon_{i}\left(\Gamma_{i}^{-1} s\right), \quad s \in \mathbb{R}_{+},
$$

where $\left\{\varepsilon_{i}\right\}_{i \in \mathbb{N}}$ are i.i.d. copies of $\varepsilon(s), s \in \mathbb{R}_{+}$, such that

$$
\mathbb{E} \int_{0}^{\infty} \min \left(1, \varepsilon(s)^{2}\right) s^{-2} d s<\infty
$$

because of (3.7). 
The setting can be altered by considering the family of non-negative càdlàg functions with the identical involution. In particular, each seperable in probability càdlàg time-stable process with non-negative values admits a LePage series representation.

Example 4.6. Let $\mathbb{K}$ be the family of locally finite measures $\mu$ on $\mathbb{R}^{d}$ with the arithmetic addition, identical involution, and the Borel $\sigma$ algebra generated by the vague topology [4, Sec. 9.1]. An infinitely divisible locally finite random measure admits a Lévy measure, see [4]. A countable separating family of continuous characters consists of

$$
\chi(\mu)=\exp \left\{-\int u d \mu\right\},
$$

where $u$ belongs to an appropriately chosen countable family of continuous functions with compact support. If the scaling operation is applied to the values of measures, then $\chi(t x) \rightarrow 1$ as $t \downarrow 0$ for all $x$, so that a transversal can be constructed as in Remark 2.16. An alternative way of constructing a measurable trasversal $\mathbb{S}$ is to take a sequence $\left\{B_{k}\right\}_{k \in \mathbb{N}}$ of bounded sets that form a base of the topology and let $\mu \in \mathbb{S}$ if $\mu\left(B_{0}\right)=\cdots=\mu\left(B_{n-1}\right)=0$ and $\mu\left(B_{n}\right)=1$ for some $n$. By Theorem 3.11, each stable locally finite random measure admits the LePage representation

$$
\mu \stackrel{d}{\sim} \sum_{i \in \mathbb{N}} \Gamma_{i}^{-1 / \alpha} \varepsilon_{i}
$$

for a sequence $\left\{\varepsilon_{i}\right\}_{i \in \mathbb{N}}$ of i.i.d. locally finite measures with $\alpha$-integrable values.

Example 4.7. Let $\mathbb{K}$ be the family of closed sets $F$ in $\mathbb{R}^{d} \backslash\{0\}$ with the union as the semigroup operation, identical involution, the conventional scaling, and the $\sigma$-algebra generated by families $\{F: F \cap K \neq \varnothing\}$ for all compact sets $K$. The countable separating family of continuous characters is given by $\chi(F):=\mathbf{1}_{F \cap G=\varnothing}$ for open sets $G$ from the base of topology on $\mathbb{R}^{d}$. Note that deterministic closed sets have idempotent distributions in this cone. It is known that each union infinitely divisible random closed set admits a Lévy measure, see [19]. Thus, each strictly $\alpha$-stable random closed set $\xi$ without idempotent factors (so that $\mathbb{P}\{x \in \xi\}<1$ for all $x \in \mathbb{R}^{d}$ ) admits the series representation as the union of $\Gamma_{i}^{-1 / \alpha} \varepsilon_{i}, i \in \mathbb{N}$, for a sequence $\left\{\varepsilon_{i}\right\}_{i \in \mathbb{N}}$ of i.i.d. random closed sets.

Example 4.8. Let $\mathbb{K}$ be the family of all non-decreasing functions $\Phi: \mathcal{K} \mapsto \mathbb{R}_{+}$defined on the family $\mathcal{K}$ of compact subsets of $\mathbb{R}^{d}$ that vanish at the empty set and are upper semicontinuous, that is 
$\Phi\left(K_{n}\right) \downarrow \Phi(K)$ as $K_{n} \downarrow K$. Such functions are known as capacities (and sometimes are called topological pre-capacities, see [8]). Equip $\mathbb{K}$ with the semigroup operation by taking pointwise maximum of two capacities and the scaling of their values. It is shown in [21] that an infinitely divisible capacity $\xi$ admits a Lévy measure. It does not have idempotent factors if the essential infimum of $\xi(K)$ vanishes for all $K \in \mathcal{K}$. By Theorem 3.11, each strictly $\alpha$-stable capacity admits the series representation $\sum_{i \in \mathbb{N}} \Gamma^{-1 / \alpha} \varepsilon_{i}$, see also [20, Th. 4.1].

Example 4.9. Let $\mathbb{K}$ be the family of metric measure spaces with the Cartesian product as the semigroup operation and the scaling applied to the metric, see [9] for details. This semigroup admits a countable separating family of characters and a measurable transversal. Furthermore, each infinitely divisible random element in $\mathbb{K}$ admits a Lévy measure and so Theorem 3.11 applies and yields the LePage series representation of stable metric measure spaces obtained in [9, Th. 10.3].

\section{ACKNOWLEDGMENT}

The paper was initiated while SE was visiting the University of Bern supported by the Swiss National Science Foundation.

\section{REFERENCES}

[1] A. Araujo and E. Giné. The central limit theorem for real and Banach valued random variables. John Wiley \& Sons, New York-Chichester-Brisbane, 1980. Wiley Series in Probability and Mathematical Statistics.

[2] A. Basse-O'Connor and J. Rosiński. On the uniform convergence of random series in Skorohod space and representations of càdlàg infinitely divisible processes. Ann. Probab., 41(6):4317-4341, 2013.

[3] C. Berg, J. P. R. Christensen, and P. Ressel. Harmonic analysis on semigroups, volume 100 of Graduate Texts in Mathematics. Springer-Verlag, New York, 1984. Theory of positive definite and related functions.

[4] D. J. Daley and D. Vere-Jones. An introduction to the theory of point processes. Vol. II. Probability and its Applications (New York). Springer, New York, second edition, 2008. General theory and structure.

[5] Y. Davydov, I. Molchanov, and S. Zuyev. Strictly stable distributions on convex cones. Electron. J. Probab., 13:no. 11, 259-321, 2008.

[6] L. de Haan. A spectral representation for max-stable processes. Ann. Probab., 12(4):1194-1204, 1984.

[7] C. Dellacherie and P.-A. Meyer. Probabilities and potential, volume 29 of NorthHolland Mathematics Studies. North-Holland Publishing Co., Amsterdam-New York; North-Holland Publishing Co., Amsterdam-New York, 1978.

[8] J. L. Doob. Classical potential theory and its probabilistic counterpart. Classics in Mathematics. Springer-Verlag, Berlin, 2001. Reprint of the 1984 edition.

[9] S. N. Evans and I. Molchanov. The semigroup of metric measure spaces and its infinitely divisible measures. Trans. Amer. Math. Soc., 2015. To appear. 
[10] B. Fuchssteiner and W. Lusky. Convex cones, volume 56 of North-Holland Mathematics Studies. North-Holland Publishing Co., Amsterdam-New York, 1981. Notas de Matemática [Mathematical Notes], 82.

[11] W. N. Hudson, Z. J. Jurek, and J. A. Veeh. The symmetry group and exponents of operator stable probability measures. Ann. Probab., 14(3):1014-1023, 1986.

[12] W. N. Hudson and J. D. Mason. Operator-stable laws. J. Multivariate Anal., 11(3):434-447, 1981.

[13] Z. Kabluchko and S. Stoev. Minimal spectral representations of infinitely divisible and max-infinitely divisible processes. Bernoulli, 2015. To appear. ArXiv Math:1207.4983.

[14] E. Klein and A. C. Thompson. Theory of correspondences. Canadian Mathematical Society Series of Monographs and Advanced Texts. John Wiley \& Sons, Inc., New York, 1984. Including applications to mathematical economics, A Wiley-Interscience Publication.

[15] C. Kopp and I. Molchanov. Series representation of time-stable stochastic processes. Technical report, 2015. Arxiv Math: 1504.02969.

[16] R. LePage, M. Woodroofe, and J. Zinn. Convergence to a stable distribution via order statistics. Ann. Probab., 9(4):624-632, 1981.

[17] R. Mansuy. On processes which are infinitely divisible with respect to time. Technical report, Arxiv math:0504408, 2005.

[18] G. Maruyama. Infinitely divisible processes. Theor. Probab. Appl., 15:1-23, 1970.

[19] I. Molchanov. Theory of random sets. Probability and its Applications (New York). Springer-Verlag London, Ltd., London, 2005.

[20] I. Molchanov and K. Strokorb. Max-stable random sup-measures with comonotonic tail dependence. Technical report, 2015. Arxiv Math: 1507.03476.

[21] T. Norberg. Random capacities and their distributions. Probab. Theory Relat. Fields, 73(2):281-297, 1986.

[22] K. R. Parthasarathy. Probability measures on metric spaces. Probability and Mathematical Statistics, No. 3. Academic Press, Inc., New York-London, 1967.

[23] B. S. Rajput and J. Rosiński. Spectral representations of infinitely divisible processes. Probab. Theory Related Fields, 82(3):451-487, 1989.

[24] J. Rosiński. On path properties of certain infinitely divisible processes. Stoch. Proc. Appl., 33:73-87, 1989.

[25] J. Rosiński. On series representations of infinitely divisible random vectors. Ann. Probab., 18(1):405-430, 1990.

[26] I. Z. Ruzsa and G. J. Székely. Algebraic probability theory. Wiley Series in Probability and Mathematical Statistics: Probability and Mathematical Statistics. John Wiley \& Sons, Ltd., Chichester, 1988.

[27] G. Samorodnitsky and M. S. Taqqu. Stable non-Gaussian random processes: stochastic models with infinite variance. Chapman \& Hall, New York, 1994. MR1280932.

[28] M. Sharpe. Operator-stable probability distributions on vector groups. Trans. Amer. Math. Soc., 136:51-65, 1969. 
Department of Statistics \#3860, 367 Evans Hall, University of CaLIFORNIA, BERKELEY, CA 94720-3860, USA

E-mail address: evans@stat.berkeley.edu

University of Bern, Institute of Mathematical Statistics And ACtuarial Science, Sidlerstrasse 5, CH-3012 Bern, SWITZERLAND

E-mail address: ilya.molchanov@stat.unibe.ch 